\title{
Ghrelin receptor signaling contributes to fasting-induced suppression of conditioned avoidance behavior and neural circuit activation in male rats
}

\author{
Caitlyn M. Edwards, Tyla Dolezel, \& Linda Rinaman*
}

Florida State University, Department of Psychology, Program in Neuroscience, 1107 West Call Street, Tallahassee, FL 32304, USA

*Corresponding author. Email address: rinaman@psy.fsu.edu

Abbreviated title: Ghrelin influences passive avoidance

Number of pages: 19

Number of figures: 5

Number of words for abstract: 226

Number of words for introduction: 681

Number of words for discussion: 1117

Conflict of interest statement: The authors declare no competing financial interests.

Acknowledgments: Research reported in this paper was funded by the National Institutes of Health grants F31MH119784 (C.M.E.) and R01MH59911 (L.R.). 


\begin{abstract}
Interoceptive feedback to the brain regarding the body's physiological state plays an important role in guiding motivated behaviors. For example, a state of negative energy balance tends to increase exploratory/food-seeking behaviors while reducing avoidance behaviors. We recently reported that overnight food deprivation reduces conditioned passive avoidance behavior in male (but not female) rats. Since fasting increases circulating levels of ghrelin, we hypothesized that ghrelin signaling contributes to the observed behavioral effect of fasting. To test this, ad libitumfed male rats were trained in a passive avoidance procedure using mild footshock. Later, following overnight food deprivation, the same rats were pretreated with ghrelin receptor antagonist (GRA) or saline vehicle $30 \mathrm{~min}$ before avoidance testing. GRA restored passive avoidance in fasted rats as measured both by latency to enter and time spent in the shock-paired context. In addition, compared to vehicle-injected fasted rats, fasted rats that received GRA before re-exposure to the shock-paired context displayed more activation of prolactin-releasing peptide (PrRP)-positive noradrenergic neurons in the caudal nucleus of the solitary tract, accompanied by more activation of downstream neurons in the bed nucleus of the stria terminalis and paraventricular nucleus of the hypothalamus. These results support the view that ghrelin signaling contributes to the inhibitory effect of fasting on learned passive avoidance behavior, perhaps by suppressing recruitment of PrRP-positive noradrenergic neurons and their downstream hypothalamic and limbic forebrain targets.
\end{abstract}

\title{
Significance Statement
}

Motivated approach and avoidance behaviors are modulated by interoceptive feedback regarding physiological state. In rats, a state of energy deficit reduces avoidance of an aversively conditioned context. Here we demonstrate a role for ghrelin signaling in this effect, such that ghrelin reduces activation of noradrenergic projections from hindbrain to hypothalamus and limbic forebrain. These novel results point to a potential mechanism by which metabolic state influences learned avoidance. Improved understanding of how interoceptive feedback signals are integrated with emotional memories that drive motivated behavior should enhance development of improved therapies for anxiety and stress-related disorders. 


\section{Introduction}

Avoiding danger is critical for survival, and animals are equipped with neural circuits that enable adaptive avoidance responses to stimuli previously associated with potentially dangerous, aversive experiences. However, excessive conditioned avoidance can be maladaptive, contributing to clinical anxiety and other stress-related disorders (American Psychiatric Association, 2013; Pittig et al., 2018; Dymond, 2019). Conditioned avoidance responses are based on learning and memory processes that include both conscious and subconscious components. Sensory feedback from body to brain during the learning and expression of emotional responses plays a major role in the subconscious modulation of motivated behavior (Maniscalco and Rinaman, 2018). For example, short-term negative energy balance generally biases animals towards approach behaviors and away from avoidance behaviors, thereby facilitating exploratory food-seeking (Lutter et al., 2008; Maniscalco et al., 2015). In recent work, we demonstrated a reduction in learned passive avoidance behavior in male rats that were tested following an overnight fast (Edwards et al., 2021). The present study was designed to investigate potential neural circuits through which overnight food deprivation suppresses conditioned passive avoidance behavior.

Interoceptive feedback about the internal state of the body is continuously communicated to the brain through hormonal and neural pathways. The neural pathways feature vagal afferent inputs to the caudal nucleus of the solitary tract (cNTS) that can powerfully modulate motivated behavior (Klarer et al., 2014; Foster et al., 2017; Maniscalco and Rinaman, 2018; Holt and Rinaman, 2021). Vagal afferents communicate a diverse array of cardiovascular, respiratory, gastrointestinal, and inflammatory signals to the cNTS in a manner that is further modulated by hormonal and metabolic state (Shapiro and Miselis, 1985; Altschuler et al., 1989; Raybould, 2010; Mayer, 2011; Dockray, 2014). Within the cNTS, the A2 noradrenergic cell group receives direct synaptic input from vagal sensory afferents (Appleyard et al., 2007; Rinaman, 2011), and A2 neurons are activated to express the immediate-early gene product, cFos, in response to vagal sensory stimulation (Mönnikes et al., 1997; Rinaman, 2011; Maniscalco and Rinaman, 2013; Maniscalco et al., 2020). A caudal subset of A2 neurons that co-express prolactin-releasing peptide (PrRP) express cFos in rats exposed to a variety of innate and conditioned stressors that promote avoidance behavior (Zhu and Onaka, 2002; Morales and Sawchenko, 2003; Maniscalco et al., 2015; Edwards et al., 2021). Interestingly, overnight food deprivation markedly reduces the ability of these innate and conditioned stress stimuli to activate PrRP+ neurons within the cNTS (Maniscalco et al., 2015; Edwards et al., 2021), concurrent with reduced activation of neurons within hypothalamic and limbic forebrain regions that are innervated by PrRP+ neurons, including the paraventricular nucleus of the hypothalamus (PVN) (Maniscalco and Rinaman, 2013) and the anterior ventrolateral bed nucleus of the stria terminalis (vlBNST) (Maniscalco et al., 2015; Edwards et al., 2021).

Food deprivation increases circulating levels of ghrelin (Tschop et al., 2000; Cummings et al., 2001), and ghrelin reduces tonic firing and stimulus-induced responsiveness of vagal sensory neurons (Date et al., 2002, 2005) and postsynaptic A2 neurons in the cNTS (Cui et al., 2011). We recently demonstrated that systemic administration of a ghrelin receptor antagonist in 
fasted rats partially restores the ability of vagal sensory stimulation to activate PrRP+ A2 neurons and suppress food intake (Maniscalco et al., 2020). Further, caloric restriction reduces innate avoidance behavior in wildtype mice but not in mice lacking ghrelin receptors (Lutter et al., 2008), which display more innate anxiety-like behavior than wildtype mice (Spencer et al., 2012). Consistent with these anxiolytic effects of ghrelin signaling, baseline ghrelin levels prior to auditory fear conditioning negatively predict conditioned freezing behavior in rats (Harmatz et al., 2018). However, the potential impact of ghrelin signaling on the expression of learned avoidance has not been examined. Based on our previous research demonstrating that rats fasted overnight display less passive avoidance behavior and less fear context-induced neural activation (Edwards et al., 2021), the present study was designed to test the hypothesis that increased ghrelin receptor signaling contributes to the ability of overnight food deprivation to suppress learned passive avoidance behavior, and to suppress conditioned context-induced activation of PrRP+ neurons in the cNTS and their downstream targets in the PVN and anterior vlBNST.

\section{Methods}

All experiments were conducted in accordance with the National Institutes of Health Guide for the Care and Use of Laboratory Animals and were reviewed and approved by the Florida State University Animal Care and Use Committee.

\section{Passive Avoidance Task}

Adult male Sprague Dawley rats [Envigo; N=32 (250-300g body weight)] were pairhoused in standard tub cages in a temperature- and light-controlled housing environment (lights on from $0400 \mathrm{hr}$ to $1600 \mathrm{hr}$ ). Only male rats were used in the current study based on evidence that food deprivation does not significantly reduce conditioned passive avoidance in female rats (Edwards et al., 2021). Rats were acclimated to handling for three days, with free access to water and rat chow (Purina 5001). Rats underwent passive avoidance training in a novel light/dark shuttle box (Colbourn Instruments, Allentown, PA), with training performed 4-6 hr after lights on. The light/dark box comprised a light (illuminated) chamber with clear plastic walls and a smooth white plastic floor, and a dark (non-illuminated) chamber with black plastic walls and a metal grid floor. Each chamber measured $25 \times 25 \mathrm{~cm}$, with $28 \mathrm{~cm}$-high walls and a ceiling. The two chambers were separated by a metal divider wall with a guillotine door that could be opened and closed remotely. For passive avoidance training, rats were initially placed individually into the light chamber of the box and the dividing guillotine door was lifted immediately to allow access to the dark chamber. As expected, due to their innate aversion to the light and preference for the dark, rats very quickly entered the dark chamber (average latency $=22.73 \mathrm{~s}$ ). Upon entry into the dark chamber, the guillotine door was closed. After a 5 s delay, rats received a single mild electric footshock $(0.6 \mathrm{~mA} ; 1 \mathrm{~s})$. Rats remained in the enclosed dark chamber for $30 \mathrm{~s}$ following footshock, and then were returned to their homecage. Cagemates were similarly trained on the same day.

Two hours before dark onset on the day before testing (i.e., 2 days after training), all rats were transferred to clean cages with chow removed to initiate overnight food deprivation. On the next day, 4-6 hr after lights on, rats received either an intraperitoneal injection of $0.15 \mathrm{M} \mathrm{NaCl}$ 
(saline) vehicle $(\sim 0.3 \mathrm{ml})$, or the same volume of vehicle containing ghrelin receptor antagonist (GRA; [D-Lys ${ }^{3}$ ]-GHRP-6; 3.3mg/kg BW). This dose was based on our previous report demonstrating the ability of GRA to restore cholecystokinin-induced NTS neuronal activation in food deprived rats (Maniscalco et al., 2020). Thirty minutes later, rats were tested for passive avoidance retention. For this purpose, rats were placed individually into the light chamber of the light/dark box, the guillotine door was opened, and the latency of rats to fully enter the dark chamber was recorded, with a pre-set maximum latency of 900s (15 min). During the retention test, the guillotine door remained open and no footshock was administered. Rats were allowed to freely explore both the light and dark chambers during the 900s test, and total time spent within each chamber was recorded. After testing was complete, rats were returned to their home cages. Chow was not returned until $2 \mathrm{hr}$ prior to dark onset (i.e., approximately 6-8 hr after testing).

\section{Novel Object Recognition Task}

To examine the impact of GRA on general memory function, the same rats were tested for novel object recognition at least 5 days after completing passive avoidance testing. For this, rats $(\mathrm{N}=32)$ underwent 3 days of acclimation (10 min/day) to a previously novel tub cage that had a thin layer of bedding on the floor, with the tub enclosed in an illuminated soundattenuating chamber. On the $4^{\text {th }}$ day, rats underwent object training 4-6 hr after lights on during which two similar sized but geometrically distinct ceramic objects with rounded contours (i.e., a white pyramidal "Eiffel tower" and a blue spherical "rocket ship") were placed at opposite ends of the tub cage. Rats were allowed to freely explore both objects for 15 minutes while their behavior was recorded and later analyzed using behavior analysis software (ANY-maze, Stoelting Co.). During training, there was no significant difference between time spent with either object.

Two hours before dark onset on the day before testing (i.e., 2 days after object training), all rats were transferred to clean cages with chow removed. On the subsequent day, rats received either an i.p. injection of saline vehicle $(\sim 0.3 \mathrm{ml})$, or the same volume of vehicle containing GRA $(3.3 \mathrm{mg} / \mathrm{kg} \mathrm{BW})$. Thirty minutes later, rats were tested for novel object recognition. For this, the previously explored "Eiffel tower" object was replaced with a novel object (i.e., a grey cubeshaped "robot"). Rats were allowed to freely explore the novel and familiar objects for 15 minutes. The time each rat spent exploring each object was recorded, and the discrimination index was calculated as the difference between time spent with each object divided by the total time spent with either object: Discrimination Index $=\frac{\text { Time }_{\text {Novel }}-\text { Time }_{\text {Familiar }}}{\text { Time }_{\text {Novel }}+\text { Time }_{\text {Familiar }}}$

Time spent with an object was defined as the rat being in contact with or actively examining the object. In the discrimination index, a value of 0 indicates no discrimination between the novel and familiar objects. A positive value indicates more time spent with the novel object; whereas a negative value indicates more time spent with the familiar object. One rat from each injection group was excluded from analysis after one of the objects was knocked over during the novel object recognition test.

\section{Open Field Test}


To examine the impact of GRA on general locomotion and anxiety-like behavior, the same rats $(\mathrm{N}=32)$ were tested in the open field after overnight food deprivation. On the testing day (4-6 hr after lights on), fasted rats received either an i.p. injection of saline vehicle ( $0.3 \mathrm{ml})$, or the same volume of vehicle containing GRA $(3.3 \mathrm{mg} / \mathrm{kg} \mathrm{BW})$. Thirty minutes later, rats were placed into the center of a novel $95 \mathrm{x} 95 \mathrm{~cm}$ open arena, and then left to freely explore for $10 \mathrm{~min}$. Behavior in the open field was recorded using a Logitech webcam and was analyzed using automated behavior analysis software (ANY-maze, Stoelting Co.). Locomotion was defined as the total distance traveled during the test. Anxiety-like behaviors were interpreted based on time spent in the center zone of the field $(\sim 30 \times 30 \mathrm{~cm})$ and latency to first enter the center zone. Data from one cohort of rats ( $n=4 /$ injection group) were excluded due to lighting issues in the testing room.

\section{Context Re-exposure/Perfusions}

To examine neural activation in response to the conditioned context associated with footshock, all rats were retrained in the passive avoidance task. For this, ad libitum fed rats were once again placed into the light side of the light/dark box and received a mild electric footshock upon entry into the dark chamber, as previously described. Rats that did not enter the dark chamber within 900s were pushed in by the researcher. Two days later, 2 hours before dark onset, all rats were transferred to clean cages with chow removed to initiate overnight food deprivation. On the next day, 4-6 hr after lights on, rats received either an intraperitoneal injection of $0.15 \mathrm{M} \mathrm{NaCl}$ (saline) vehicle $(\sim 0.3 \mathrm{ml})$, or the same volume of vehicle containing GRA $(3.3 \mathrm{mg} / \mathrm{kg} \mathrm{BW})$. Thirty minutes later, rats were placed into the shock-associated dark chamber for 10 minutes with the guillotine door shut; rats were then returned to their homecage. Sixty minutes later, rats were deeply anesthetized with pentobarbital sodium $(39 \mathrm{mg} / \mathrm{ml}$ i.p., Fatal Plus Solution; Butler Schein) and transcardially perfused with saline (100mL) followed by $4 \%$ paraformaldehyde $(500 \mathrm{~mL})$.

\section{Histology}

Fixed brains were removed from the skull, post-fixed overnight at $4{ }^{\circ} \mathrm{C}$, then cryoprotected in $20 \%$ sucrose. Brains were blocked and sectioned coronally ( $35 \mu \mathrm{m}$ ) using a Leica freezing-stage sliding microscope. Tissue sections were collected in six serial sets and stored at $-20^{\circ} \mathrm{C}$ in cryopreservant solution (Watson et al., 1986) until immunohistochemical processing. Primary and secondary antisera were diluted in $0.1 \mathrm{M}$ phosphate buffer containing $0.3 \%$ Triton X-100 and 1\% normal donkey serum. One set of tissue sections from each rat (with each set containing a complete rostrocaudal series of sections spaced by $210 \mu \mathrm{m}$ ) was incubated in a rabbit polyclonal antiserum against cFos (1:10,000; Cell Signaling, 2250; AB_2247211), followed by biotinylated donkey anti-rabbit IgG (1:500; Jackson ImmunoResearch). Sections were then treated with Elite Vectastain ABC reagents (Vector) and reacted with diaminobenzidine $(\mathrm{DAB})$ intensified with nickel sulfate to produce a blue-black nuclear cFos reaction product.

To visualize cFos within hindbrain PrRP neurons, one set of cFos-labeled tissue sections from each rat was subsequently incubated in a rabbit polyclonal antiserum against PrRP 
(1:10,000; Phoenix Pharmaceuticals, H-008-52), followed by biotinylated donkey anti-rabbit IgG (1:500; Jackson ImmunoResearch) and Elite Vectastain ABC reagents (Vector), and finally reacted with plain $\mathrm{DAB}$ to produce a brown cytoplasmic reaction product.

To visualize cFos within all noradrenergic neurons, a second set of tissue from each rat was incubated in a cocktail of rabbit anti-cFos (1:1000) and a mouse monoclonal antiserum against dopamine beta-hydroxylase (DbH; 1:5000; Millipore, MAB308; AB_2245740), followed by a cocktail of Cy3-conjugated donkey anti-rabbit IgG (red) and Alexa Fluor 488-conjugated donkey anti-mouse IgG (green).

\section{Quantification of Neural Activation}

Sections through the cNTS ( 15.46-13.15 mm caudal to bregma) were imaged at 20x using a Keyence microscope (BZ-X700). Using separate sets of dual-labeled tissue sections from each rat, the total numbers of $\mathrm{DbH}+$ and PrRP+ cNTS neurons were counted, and the percentage of each chemically identified neural population co-expressing cFos was determined. A neuron was considered to be cFos-positive if its nucleus contained any visible cFos immunolabeling.

Sections through the anterior vlBNST $(\sim 0.26-0.40 \mathrm{~mm}$ caudal to bregma) were imaged at 10x. Images from two rats were excluded from further analysis due to poor tissue quality. Using ImageJ, a circular region of interest (ROI) of $0.10 \mathrm{~mm}^{2}$ was centered on the area containing the highest density of $\mathrm{DbH}+$ fibers and terminals corresponding to the fusiform subnucleus of the anterior vlBNST (Maruyama et al., 1999; Lin et al., 2002; Maniscalco et al., 2015). The number of cFos-positive neurons within each $0.10 \mathrm{~mm}^{2}$ ROI was determined bilaterally in 2-3 sections per brain and averaged across assessed ROIs in each animal.

Three sections through the PVN ( 1.5-1.9mm caudal to bregma) were imaged at 10x bilaterally per brain. A region of interest (ROI) encompassing the $\mathrm{DbH}+$ fibers and terminals was drawn using ImageJ and the number of cFos-positive neurons within each ROI was determined, divided by the area of the ROI, and averaged across assessed ROIs in each animal to produce the number of cFos-positive neurons per $\mathrm{mm}^{2}$.

\section{Statistics}

Data were analyzed using GraphPad Prism. Passive avoidance behaviors (latency to enter and total time spent in the dark chamber) during the retention test were analyzed using a Welch's t-test (Ruxton, 2006). Time spent exploring novel vs. familiar objects in the novel object recognition task was analyzed with a mixed 2x2 ANOVA with i.p. injection as a betweensubjects independent variable and Object (novel, familiar) as a within-subjects independent variable. When ANOVA F values indicated significant main effects and/or interactions, post-hoc comparisons were made using Š́dák's multiple comparisons tests. Discrimination indices for novel object recognition by each i.p. injection group of rats were analyzed using Welch's t-test, and by individual one-subject t-tests with a hypothetical value of 0 (i.e., no discrimination). Each open field behavioral measure (distance traveled, time in center, and latency to first enter the center) was analyzed separately with a Welch's t-test to compare i.p. injection groups. Cell count data (cFos activation of PrRP and DbH neurons, cFos activation within the anterior vlBNST and 
PVN) were analyzed using Welch's t-test. In addition, Pearson's r correlations were run between the percent of $\mathrm{DbH}+/ \mathrm{PrRP}+\mathrm{cNTS}$ neurons expressing cFos and cFos counts within vlBNST/PVN in the same rat. An alpha level of $0.05(\mathrm{p} \leq 0.05)$ was used as the criterion for considering group differences to be statistically significant. Estimation statistics were used to report effect sizes for both passive avoidance behavior and cell count data (Calin-Jageman and Cumming, 2019; Ho et al., 2019).

\section{Results}

\section{Passive Avoidance Task}

Welch's t-test indicated that overnight food deprived rats injected with GRA demonstrated more passive avoidance behavior than food deprived rats injected with saline. GRA-treated rats displayed faster entry into the shock-paired dark chamber $[\mathrm{t}(26.1)=1.728 ; \mathrm{p}=$ 0.0479], and increased time spent in the dark chamber [t(18.67) $=2.162 ; \mathrm{p}=0.0219$ ] (Fig. 1). Examining the unpaired mean difference between saline and GRA treatment using estimation statistics indicated food deprived rats pretreated with GRA took an average of $195 \mathrm{~s}$ (3.25min) longer to enter the shock-paired dark chamber compared to saline-injected controls [95\% CI $11.4 \mathrm{~s}, 419 \mathrm{~s}]$, and spent an average of $178 \mathrm{~s}(2.97 \mathrm{~min})$ less in the shock-paired dark chamber during the 900 s retention test [95\%CI 47.2s, 369s].

\section{Novel Object Recognition Task}

GRA treatment did not alter the behavior of fasted rats in the novel object recognition task (Fig. 2). Two-way ANOVA indicated a significant main effect of Object $[\mathrm{F}(1,28)=15.59 ; \mathrm{p}$ $=0.0005$ ] but no main effect of i.p. treatment and no interaction. Post-hoc Sídák's multiple comparisons tests indicated that fasted rats spent significantly more time exploring the novel object compared to the familiar object in both the saline- $(\mathrm{p}=0.0093)$ and GRA-injected $(\mathrm{p}=$ 0.0361) groups. Individual one-sample t-tests indicated that the discrimination indices for both the saline- $[\mathrm{t}(14)=3.575, \mathrm{p}=0.0030]$ and GRA-injected groups $[\mathrm{t}(14)=2.294, \mathrm{p}=0.0378]$ were significantly higher than 0 , evidence that both groups were similarly able to discriminate between the familar and novel objects.

\section{Open Field Test}

Pre-treatment of fasted rats with GRA did not influence their behavior in the 10-min open field test (Fig. 3). There were no significant differences in locomotor behavior (i.e. total distance traveled) between saline-injected $($ mean $=17.9 \mathrm{~m})$ vs. GRA-injected (mean $=17.3 \mathrm{~m})$ rats (Fig. 3A). Additionally, there were no significant group differences in anxiety-like behaviors as measured by either time spend in the center (Fig. 3B) or latency to first enter the central zone of the open field (Fig. 3C).

\section{Neural Activation after Re-Exposure to the Shock-Paired Dark Chamber}

Welch's t-test indicated that food deprived rats injected with GRA before inescapable reexposure to the shock-paired dark chamber demonstrated more activation of PrRP+ neurons compared to food deprived rats injected with saline before re-exposure, as measured by the 
proportion of PrRP+ neurons co-expressing cFos $[\mathrm{t}(17.78)=2.364 ; \mathrm{p}=0.0297]$ (Fig. 4A). Examining the unpaired mean difference between saline and GRA treatment using estimation statistics indicated that $12.1 \%$ more PrRP+ neurons were activated in food deprived rats pretreated with GRA compared to saline [95\%CI 2.39\%, 21.9\%]. A similar effect of GRA pretreatment was evident in the ability of dark chamber re-exposure to activate cFos expression in the larger population of $\mathrm{DbH}+\mathrm{A} 2$ neurons $[\mathrm{t}(21.17)=2.439 ; \mathrm{p}=0.0236]$ (Fig. 4D). Examining the unpaired mean difference between saline and GRA treatment using estimation statistics indicated that $11.9 \%$ more $\mathrm{DbH}+$ neurons were activated in food deprived rats pretreated with GRA compared to saline [95\%CI 1.59\%, 20.4\%].

Regarding activation of neurons within the downstream hypothalamic and limbic forebrain targets of A2 neurons, Welch's t-test indicated that food deprived rats injected with GRA displayed more cFos activation within the anterior vlBNST compared to food deprived rats injected with saline before re-exposure to the shock-paired context $[\mathrm{t}(17.53)=2.196 ; \mathrm{p}=0.0418]$ (Fig. 5A). Examining the unpaired mean difference between saline and GRA treatment using estimation statistics indicated that 12.9 more vlBNST neurons per ROI were activated in food deprived rats pretreated with GRA compared to saline [95\%CI 3.4, 25.3]. A similar effect of GRA treatment on cFos activation was observed in the PVN $[\mathrm{t}(21.76)=2.092 ; \mathrm{p}=0.0483]$ (Fig. 5D). Examining the unpaired mean difference between saline and GRA treatment using estimation statistics indicated that 74.6 more PVN neurons $/ \mathrm{mm}^{2}$ were activated in food deprived rats pretreated with GRA compared to saline [95\%CI 8.64, 137]. Pearson's r correlations indicated a strong positive relationship between activation of $\mathrm{DbH}+\mathrm{cNTS}$ neurons and activation of neurons in the vlBNST $[\mathrm{r}(20)=0.5093 ; \mathrm{p}=0.0155]$ (Fig. 6A) and PVN $[\mathrm{r}(22)=0.6494 ; \mathrm{p}=$ 0.0006] (Fig. 6B) within the same rat. Similar positive relationships were observed between activation of PrRP+ cNTS neurons and activation of neurons in the vlBNST $[\mathrm{r}(20)=0.5497 ; \mathrm{p}=$ 0.0080] (Fig. 6C) and PVN [r(22) = 0.5488; $\mathrm{p}=0.0055]$ (Fig. 6D).

\section{Discussion}

Results from the present study build on our previous findings that overnight food deprivation reduces conditioned passive avoidance behavior and also reduces conditioned context-induced activation of PrRP+ NA neurons in the NTS and downstream target neurons in the vlBNST (Edwards et al., 2021). Here we demonstrate a role for ghrelin signaling in these fasting-induced behavioral and neural effects. Specifically, we show that systemic administration of a ghrelin receptor antagonist prior to the retention test in male food-deprived rats restores conditioned passive avoidance behavior (Fig. 1) and neuronal activation (Fig. 4+5). These novel findings support the view that reduced avoidance behavior and neural activation in food-deprived rats is due to elevated ghrelin signaling.

We considered the possibility that reduced passive avoidance behavior in food-deprived rats with elevated ghrelin signaling is due to a general reduction in memory retrieval, such that GRA may increase passive avoidance by improving memory of the shock-associated context. However, we found that GRA treatment did not improve novel object recognition memory in food deprived rats (Fig. 2). A key distinction between mnemonic processes underlying passive avoidance vs. novel object recognition is that the former but not the latter involves contextual 
memory (Atucha and Roozendaal, 2015; Lueptow, 2017). Previous research has shown that mice with a global ghrelin receptor knockout and rats with a vagal afferent-specific ghrelin receptor knockdown show impairment in a hippocampal-dependent "novel object in context" task (Diano et al., 2006; Davis et al., 2020). However, GRA effects on passive avoidance memory in the present study were in the opposite direction, such that GRA treatment restored/improved avoidance memory in food-deprived rats. This discrepancy may be related to chronic rather than acute disruption of ghrelin signaling in the knockout/knockdown studies, which could interfere with other stages of memory formation. Alternatively, reduced performance in the "novel object in context" task by rodents with ghrelin receptor knockout/knockdown could reflect avoidance of the novel object. For example, Davis and colleagues (2020) reported a negative shift in discrimination index in their vagal afferent ghrelin receptor knockdown model, such that knockdown rats spent less time exploring the novel object than would be expected if their behavior was due only to impaired memory of the familiar object. If a fasting-induced elevation in ghrelin signaling is normally anxiolytic, then GRA treatment may reverse this effect to restore general/innate anxiety-like behavior and avoidance displayed by non-fasted rats. Supporting this idea, previous work demonstrated that overnight food deprivation suppresses innate anxiety-like behavior in the elevated plus maze and light-enhanced acoustic startle tests (Maniscalco et al., 2015), and in the open field test (Heiderstadt et al., 2000). In the present study, GRA treatment did not alter innate anxiety-like behavior displayed by food-deprived rats in the open field test (Fig. 3B+C), evidence that ghrelin signaling alone cannot fully explain the anxiolytic effect of food deprivation. A previous study reported that vagal afferent-specific ghrelin receptor knockdown does not alter anxiety-like behavior in the elevated zero maze (Davis et al., 2020), although rats in that experiment were not food deprived so endogenous ghrelin presumably would have been relatively low in both knockdown and control rats.

Our findings demonstrate that GRA treatment restored the ability of re-exposure to the shock-associated context to activate cFos expression in PrRP+ noradrenergic neurons in food deprived rats (Fig. 4). These results are consistent with evidence that ghrelin suppresses the activity of vagal sensory afferents (Date et al., 2002, 2005), and suppresses activation of postsynaptic A2 neurons in the cNTS (Cui et al., 2011). Our current results also are in line with our previous report that GRA restores the ability of pharmacological vagal afferent stimulation to activate PrRP+ A2 neurons (Maniscalco et al., 2020). In that study (Maniscalco et al., 2020) and in the current study, GRA treatment did not completely restore A2 neural activation to levels observed in ad libitum fed rats [i.e., see (Edwards et al., 2021)]. Thus, elevated ghrelin signaling during food deprivation contributes to but does not fully explain the A2 neural "silencing" effect of negative energy balance. Other contributing factors may include altered mechanosensory signals and/or hormonal signals (Dallman et al., 1999; Maniscalco et al., 2015; Maniscalco \& Rinaman, 2017).

In addition to partial restoration of cNTS neural activation in GRA-treated rats after exposure to the shock-paired context, GRA increased context-induced activation of neurons within the vlBNST and PVN, two downstream targets of PrRP+ noradrenergic cNTS neurons (Fig. 5). We hypothesize that the cNTS is driving vlBNST and PVN activation, based on significant positive relationships between activation of $\mathrm{PrRP}+$ and $\mathrm{DbH}+\mathrm{cNTS}$ neurons and 
activation of neurons within the vlBNST and PVN (Fig. 6). We previously demonstrated that A2 noradrenergic inputs to the vIBNST and PVN are necessary for stress-induced innate avoidance and behavioral suppression (Banihashemi and Rinaman, 2006; Rinaman and Dzmura, 2007; Zheng and Rinaman, 2013), and noradrenergic signaling in the BNST is implicated in avoidance behavior conditioned by aversive opiate withdrawal (Aston-Jones et al., 1999; Delfs et al., 2000). The current results provide new evidence that A2 noradrenergic inputs to the vlBNST and/or PVN also participate in conditioned passive avoidance behavior.

The effect of GRA treatment on behavior and neural activation in the present study could reflect interference with either central or peripheral ghrelin signaling mechanisms. Ghrelin receptor mRNA is expressed by vagal sensory neurons in the nodose ganglia (Date et al., 2002; Burdyga et al., 2006) and by neurons within the cNTS (Lin et al., 2004; Zigman et al., 2006), PVN (Zigman et al., 2006), and hippocampus (Guan et al., 1997; Zigman et al., 2006). Since systemic administration of the same GRA used in the present study was shown to block the effects of centrally-administered ghrelin (Brockway et al., 2016), central receptor blockade may have contributed to the behavioral and neural effects of GRA in our experiments. Additional work will be needed to differentiate between peripheral/vagally-mediated vs. centrally-mediated ghrelin signaling in conditioned avoidance behavior. Future research also should explore sex differences in passive avoidance behavior and the role of ghrelin signaling (and other interoceptive feedback signals) in that behavior, given our previous report that food deprivation suppresses passive avoidance in male, but not female, rats (Edwards et al., 2021).

In summary, our results demonstrate a role for endogenous ghrelin signaling in the ability of negative energy balance to reduce conditioned avoidance behavior in male rats. We further demonstrate that GRA treatment restores the ability of a footshock-conditioned context to activate PrRP+ A2 noradrenergic neurons, and to activate neurons in the downstream targets of these cNTS neurons (i.e., vlBNST and PVN) in food deprived male rats. These findings support the view that interoceptive feedback from body to brain modulates learned avoidance behavior, with a potential role for a ghrelin-sensitive vagal afferent $\rightarrow$ NTS $\rightarrow$ vlBNST/PVN circuit in this modulatory effect. 
Figures
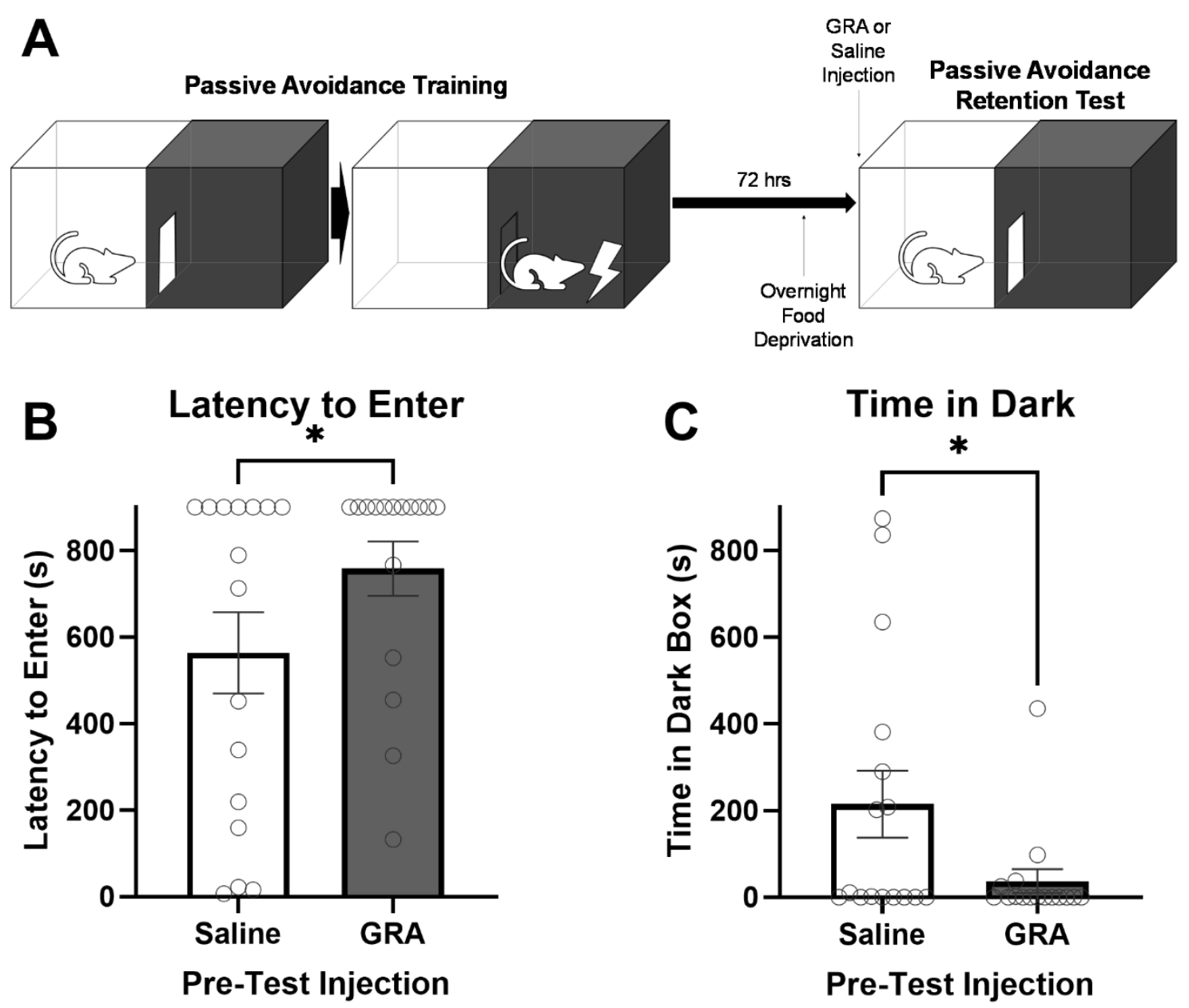

Figure 1: Systemic ghrelin receptor antagonist (GRA) administration prior to the retention test increased passive avoidance behavior in food deprived male rats. (A) A schematic depicting the passive avoidance experimental timeline. (B) Fasted rats pretreated with GRA took an average of 195s longer to enter the shock-paired dark chamber compared to fasted rats pretreated with saline. (C) Fasted rats pretreated with GRA spent an average of 178s less in the shock-paired dark chamber during the 900 s retention test compared to fasted rats pretreated with saline. ${ }^{*} \mathrm{p}<$ 0.05. Graphs display group mean +/- SEM, with individual data points plotted. 

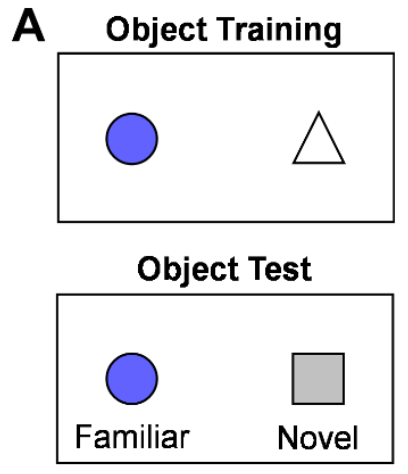

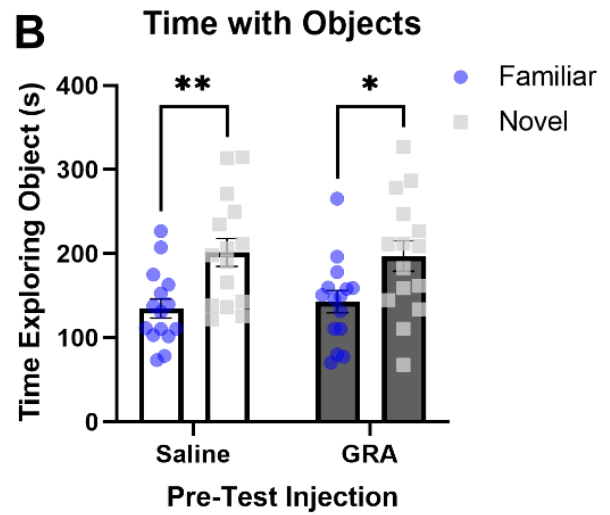

C Discrimination Index

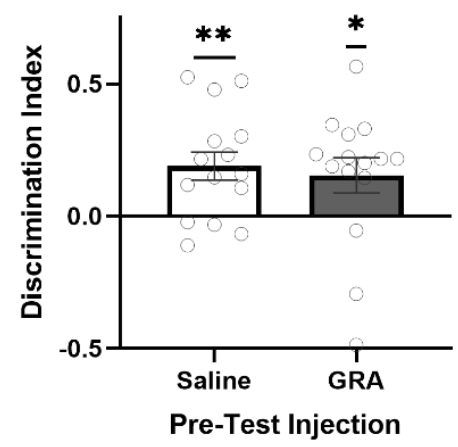

Figure 2: Systemic ghrelin receptor antagonist (GRA) administration prior to the novel object test did not influence novel object recognition memory in food deprived male rats. (A) A schematic depicting the novel object recognition training and test. (B) Both GRA- and salinepretreated food deprived rats spent significantly more time with the novel object compared to the familiar object. (C) The discrimination index for both GRA- and saline-pretreated food deprived rats were similar and significantly greater than zero. ${ }^{*} \mathrm{p}<0.05$ and ${ }^{*} \mathrm{p} p<0.01$
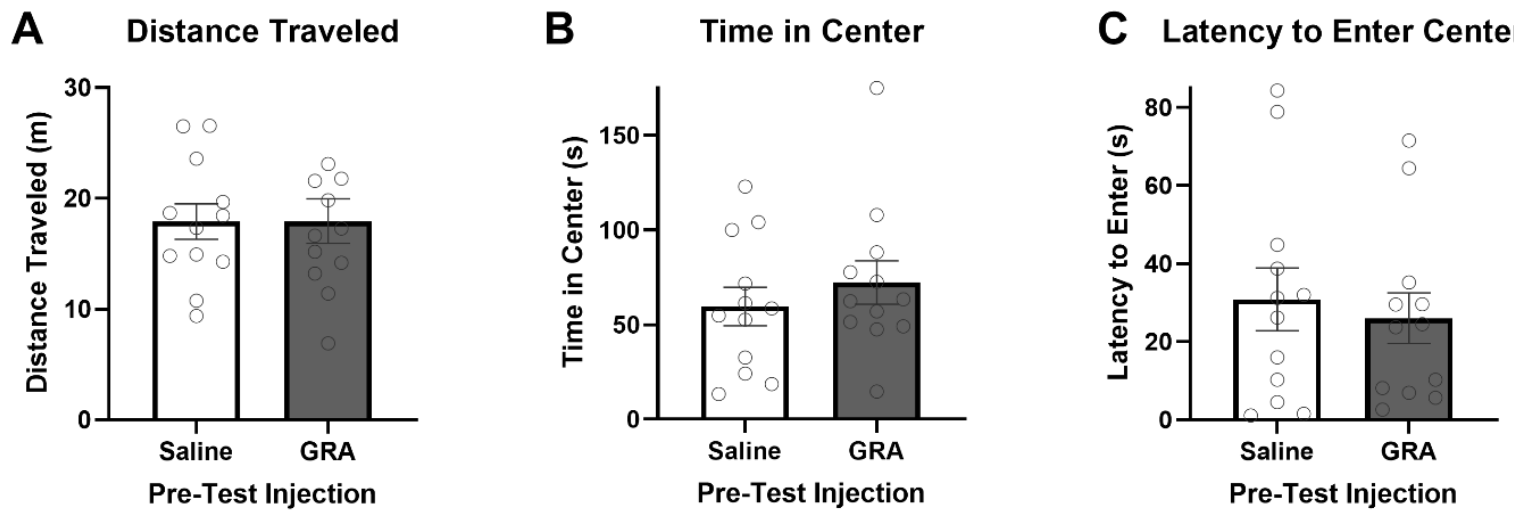

Figure 3: Systemic ghrelin receptor antagonist (GRA) administration prior to the open field test did not influence locomotion or anxiety-like behavior in food deprived male rats. (A) GRA- and saline-pretreated rats did not differ in the distance traveled during the open field test. (B) GRAand saline- pretreated rats did not differ in the time spent in the center zone of the open field test. (C) GRA- and saline-pretreated rats did not differ in the latency to enter the center zone in the open field test. 

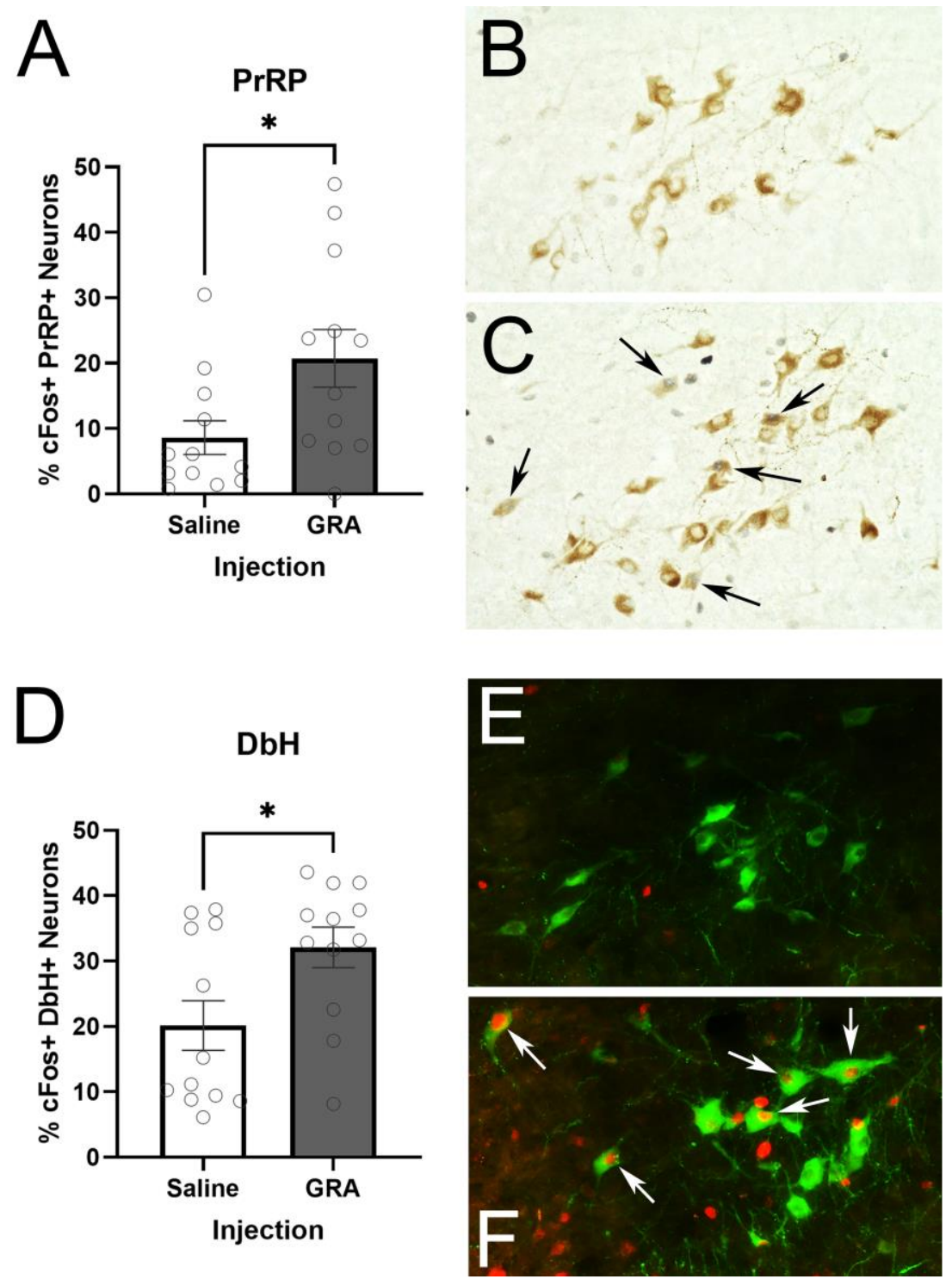

Figure 4: Systemic ghrelin receptor antagonist (GRA) administration prior to shock-paired dark chamber re-exposure increased cFos expression in noradrenergic cNTS neurons in food deprived male rats. (A) GRA pretreatment increases conditioned dark chamber-induced activation of PrRP+ neurons. $(\mathrm{B}+\mathrm{C})$ Representative images taken at 40x magnification of nuclear cFos (dark blue) and cytoplasmic PrRP immunolabeling (brown) in a saline control (B) and GRA pretreated rat (C). Black arrows indicate double-labeled (cFos+) PrRP+ cells. (D) GRA pretreatment increases conditioned dark chamber-induced activation of the broader population of $\mathrm{DbH}+\mathrm{A} 2$ neurons. $(\mathrm{E}+\mathrm{F})$ Representative images taken at 40x magnification of nuclear cFos (red) and cytoplasmic DbH immunolabeling (green) in a saline control (E) and GRA pretreated rat (F). White arrows indicate double-labeled (cFos+) DbH+ cells. ${ }^{*} \mathrm{p}<0.05$. 

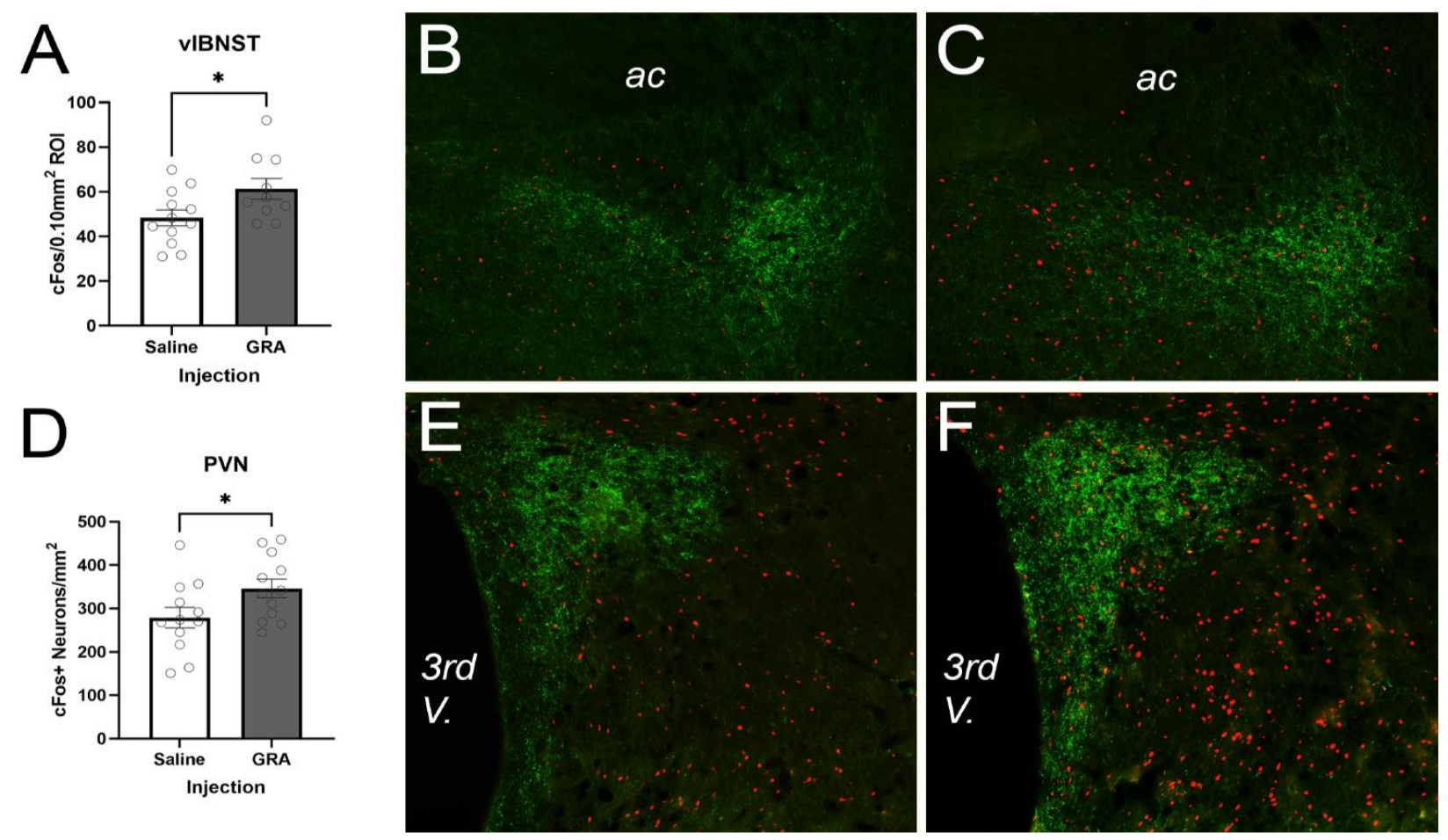

Figure 5: Systemic ghrelin receptor antagonist (GRA) administration prior to shock-paired dark chamber re-exposure increased cFos expression in neurons in the $\mathrm{DbH}+$ terminal-rich regions of vlBNST and PVN in food deprived male rats. (A) GRA pretreatment increases conditioned dark chamber-induced activation of neurons in the $\mathrm{DbH}+$ terminal-rich region of vlBNST. $(\mathrm{B}+\mathrm{C})$ Representative images of nuclear cFos (red) and $\mathrm{DbH}+$ terminal labeling (green) in a saline control (B) and GRA pretreated rat (C). ac, anterior commissure. (D) GRA pretreatment increases conditioned dark chamber-induced activation of neurons in the $\mathrm{DbH}+$ terminal-rich region of PVN. $(\mathrm{E}+\mathrm{F})$ Representative images of nuclear $\mathrm{cFos}(\mathrm{red})$ and $\mathrm{DbH}+$ terminal labeling (green) in a saline control (E) and GRA pretreated rat $(\mathrm{F}) .3^{\text {rd }} \mathrm{V}$., third ventricle. ${ }^{*} \mathrm{p}<0.05$. 
A

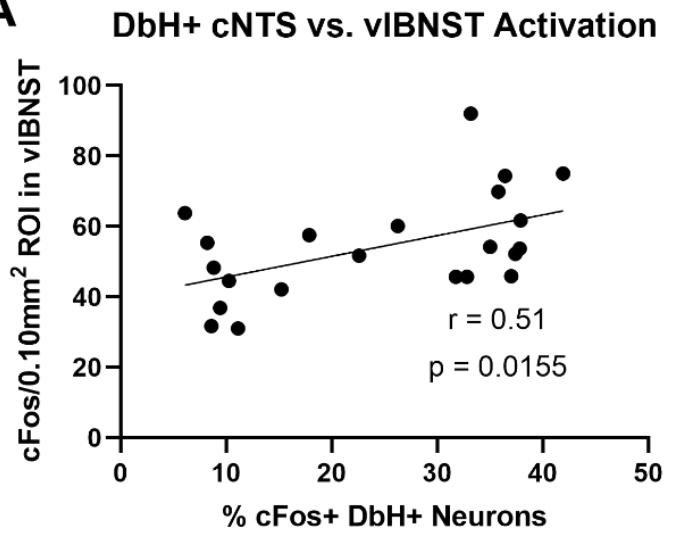

C

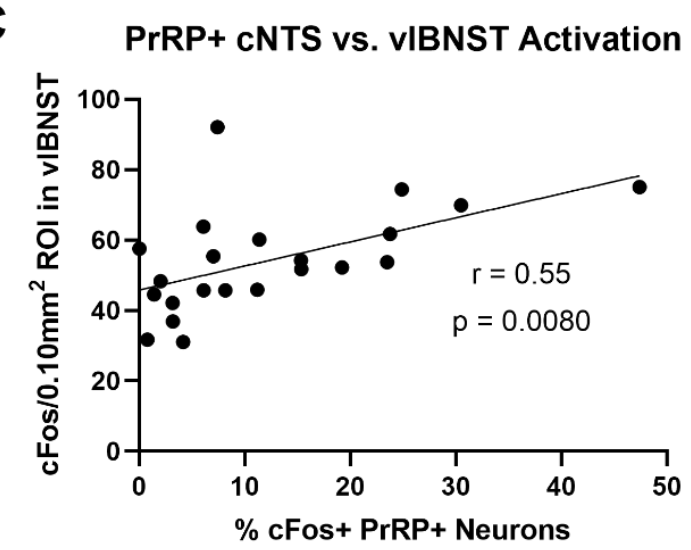

B

DbH+ cNTS vs. PVN Activation

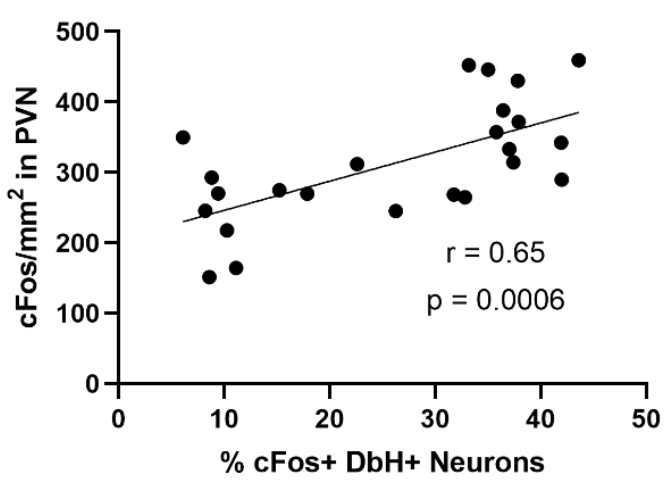

D

D PrRP+ cNTS vs. PVN Activation

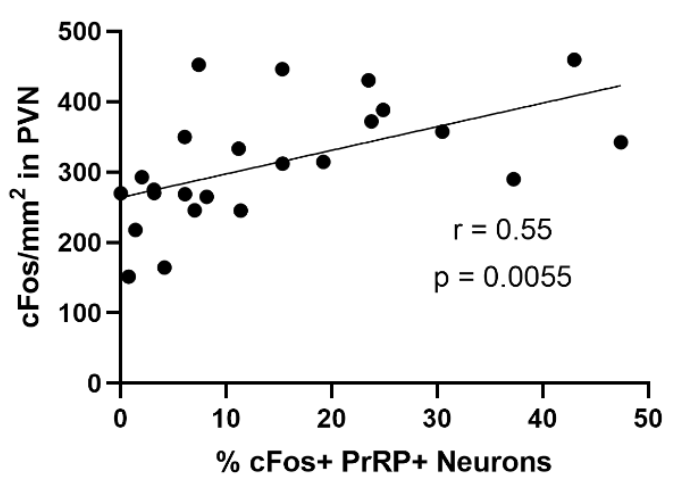

Figure 6: Scatterplots depicting significant positive relationships between dark chamber reexposure-induced activation of (A) DbH+ neurons in the cNTS and neurons in the vlBNST, (B) $\mathrm{DbH}+$ neurons and neurons in the PVN, (C) PrRP+ neurons in the cNTS and neurons in the vlBNST, and (D) PrRP+ neurons and neurons in the PVN. 


\section{References}

Altschuler SM, Bao X, Bieger D, Hopkins DA, Miselis RR (1989) Viscerotopic representation of the upper alimentary tract in the rat: Sensory ganglia and nuclei of the solitary and spinal trigeminal tracts. J Comp Neurol 283:248-268.

American Psychiatric Association (2013) Diagnostic and Statistical Manual of Mental Disorders: DSM 5. Arlington, VA: American Psychiatric Publishing.

Appleyard SM, Marks D, Kobayashi K, Okano H, Low MJ, Andresen MC (2007) Visceral afferents directly activate catecholamine neurons in the solitary tract nucleus. J Neurosci 27:13292-13302.

Aston-Jones G, Delfs JM, Druhan J, Zhu Y (1999) The bed nucleus of the stria terminalis. A target site for noradrenergic actions in opiate withdrawal. Ann N Y Acad Sci 877:486-498.

Atucha E, Roozendaal B (2015) The inhibitory avoidance discrimination task to investigate accuracy of memory. Front Behav Neurosci 9:1-8.

Banihashemi L, Rinaman L (2006) Noradrenergic inputs to the bed nucleus of the stria terminalis and paraventricular nucleus of the hypothalamus underlie hypothalamic-pituitary- adrenal axis but not hypophagic or conditioned avoidance responses to systemic yohimbine. $\mathrm{J}$ Neurosci 26:11442-11453.

Brockway ET, Krater KR, Selva JA, Wauson SER, Currie PJ (2016) Impact of [d-Lys3]-GHRP6 and feeding status on hypothalamic ghrelin-induced stress activation. Peptides 79:95-102 Available at: http://dx.doi.org/10.1016/j.peptides.2016.03.013.

Burdyga G, Varro A, Dimaline R, Thompson DG, Dockray GJ (2006) Ghrelin receptors in rat and human nodose ganglia: Putative role in regulating CB-1 and MCH receptor abundance. Am J Physiol - Gastrointest Liver Physiol 290:1289-1297.

Calin-Jageman R, Cumming G (2019) Estimation for Better Inference in Neuroscience. eNeuro $6: 1-11$.

Cui RJ, Li X, Appleyard SM (2011) Ghrelin inhibits visceral afferent activation of catecholamine neurons in the solitary tract nucleus. 76:211-220.

Cummings DE, Purnell JQ, Frayo RS, Schmidova K, Wisse BE, Weigle DS (2001) A preprandial rise in plasma ghrelin levels suggests a role in meal inititation in humans. Diabetes 50:1714-1719.

Dallman MF, Akana SF, Bhatnagar S, Bell ME, Choi SJ, Chu A, Horsley C, Levin N, Meijer O, Soriano LR, Strack AM, Viau V (1999) Starvation: Early signals, sensors, and sequelae. Endocrinology 140:4015-4023.

Date Y, Murakami N, Toshinai K, Matsukura S, Niijima A, Matsuo H, Kangawa K, Nakazato M (2002) The role of the gastric afferent vagal nerve in Ghrelin-induced feeding and growth hormone secretion in rats. Gastroenterology 123:1120-1128.

Date Y, Toshinai K, Koda S, Miyazato M, Shimbara T, Tsuruta T, Niijima A, Kangawa K, Nakazato M (2005) Peripheral interaction of ghrelin with cholecystokinin on feeding 
regulation. Endocrinology 146:3518-3525.

Davis EA, Wald HS, Suarez AN, Zubcevic J, Liu CM, Cortella AM, Kamitakahara AK, Polson JW, Arnold M, Grill HJ, de Lartigue G, Kanoski SE (2020) Ghrelin Signaling Affects Feeding Behavior, Metabolism, and Memory through the Vagus Nerve. Curr Biol 30:45104518.e6 Available at: https://doi.org/10.1016/j.cub.2020.08.069.

Delfs JM, Zhu Y, Druhan JP, Aston-Jones G (2000) Noradrenaline in the ventral forebrain is critical for opiate withdrawal-induced aversion. 403.

Diano S, Farr SA, Benoit SC, McNay EC, Da Silva I, Horvath B, Gaskin FS, Nonaka N, Jaeger LB, Banks WA, Morley JE, Pinto S, Sherwin RS, Xu L, Yamada KA, Sleeman MW, Tschöp MH, Horvath TL (2006) Ghrelin controls hippocampal spine synapse density and memory performance. Nat Neurosci 9:381-388.

Dockray GJ (2014) Gastrointestinal hormones and the dialogue between gut and brain. J Physiol 592:2927-2941.

Dymond S (2019) Overcoming avoidance in anxiety disorders: The contributions of Pavlovian and operant avoidance extinction methods. Neurosci Biobehav Rev 98:61-70 Available at: https://doi.org/10.1016/j.neubiorev.2019.01.007.

Edwards CM, Dolezel T, Rinaman L (2021) Sex and metabolic state interact to influence expression of passive avoidance memory in rats: Potential contribution of A2 noradrenergic neurons. Physiol Behav 239:113511 Available at: https://doi.org/10.1016/j.physbeh.2021.113511.

Foster JA, Rinaman L, Cryan JF (2017) Stress \& the gut-brain axis: Regulation by the microbiome. Neurobiol Stress 7:124-136 Available at: https://doi.org/10.1016/j.ynstr.2017.03.001.

Guan XM, Yu H, Palyha OC, McKee KK, Feighner SD, Sirinathsinghji DJS, Smith RG, Van Der Ploeg LHT, Howard AD (1997) Distribution of mRNA encoding the growth hormone secretagogue receptor in brain and peripheral tissues. Mol Brain Res 48:23-29.

Harmatz ES, Stone L, Lim SH, Lee G, Mcgrath A, Peng X, Kosoy E, Yao J, Liu E, Machado NJ, Weiner VS, Slocum W, Cunha RA, Goosens KA (2018) Central ghrelin resistance permits the overconsolidation of fear memory. Biol Psychiatry 81:1003-1013.

Heiderstadt KM, McLaughlin RM, Wright DC, Walker SE, Gomez-Sanchez CE (2000) The effect of chronic food and water restriction on open-field behaviour and serum corticosterone levels in rats. Lab Anim 34:20-28.

Ho J, Tumkaya T, Aryal S, Choi H, Claridge-Chang A (2019) Moving beyond P values: data analysis with estimation graphics. Nat Methods 16:565-566 Available at: http://dx.doi.org/10.1038/s41592-019-0470-3.

Holt MK, Rinaman L (2021) The role of nucleus of the solitary tract glucagon-like peptide-1 and prolactin-releasing peptide neurons in stress: anatomy, physiology and cellular interactions. Br J Pharmacol:1-17.

Klarer M, Arnold M, Gunther L, Winter C, Langhans W, Meyer U (2014) Gut Vagal Afferents 
Differentially Modulate Innate Anxiety and Learned Fear. J Neurosci 34:7067-7076 Available at: http://www.jneurosci.org/cgi/doi/10.1523/JNEUROSCI.0252-14.2014.

Lin SHS, Leslie FM, Civelli O (2002) Neurochemical properties of the prolactin releasing peptide (PrRP) receptor expressing neurons: Evidence for a role of PrRP as a regulator of stress and nociception. Brain Res 952:15-30.

Lin Y, Matsumura K, Fukuhara M, Kagiyama S, Fujii K, Iida M (2004) Ghrelin Acts at the Nucleus of the Solitary Tract to Decrease Arterial Pressure in Rats. Hypertension 43:977982.

Lueptow LM (2017) Novel object recognition test for the investigation of learning and memory in mice. $\mathrm{J}$ Vis $\operatorname{Exp} 2017: 1-9$.

Lutter M, Sakata I, Osborne-lawrence S, Rovinsky SA, Jason G, Jung S, Birnbaum S, Yanagisawa M, Elmquist JK, Nestler EJ, Zigman JM (2008) The orexigenic hormone ghrelin defends against depressive symptoms of chronic stress. Nat Neurosci 11:752-753.

Maniscalco JW, Edwards CM, Rinaman L (2020) Ghrelin signaling contributes to fastinginduced attenuation of hindbrain neural activation and hypophagic responses to systemic cholecystokinin in rats. Am J Physiol - Regul Integr Comp Physiol 318:R1014-R1023.

Maniscalco JW, Rinaman L (2013) Overnight food deprivation markedly attenuates hindbrain noradrenergic, glucagon-like peptide-1, and hypothalamic neural responses to exogenous cholecysotkinin in male rats. Physiol Behav 121:35-42 Available at: https://www.ncbi.nlm.nih.gov/pmc/articles/PMC3624763/pdf/nihms412728.pdf.

Maniscalco JW, Rinaman L (2017) Interoceptive modulation of neuroendocrine, emotional, and hypophagic responses to stress. Physiol Behav 176:1-29.

Maniscalco JW, Rinaman L (2018) Vagal Interoceptive Modulation of Motivated Behavior. Physiology 33:151-167 Available at: http://www.physiology.org/doi/10.1152/physiol.00036.2017.

Maniscalco JW, Zheng H, Gordon PJ, Rinaman L (2015) Negative energy balance blocks neural and behavioral responses to acute stress by "silencing" central glucagon-like peptide 1 signaling in rats. J Neurosci 35:10701-10714.

Maruyama M, Matsumoto H, Fujiwara K, Kitada C, Hinuma S, Onda H, Fujino M, Inoue K (1999) Immunocytochemical localization of prolactin-releasing peptide in the rat brain. Endocrinology 140:2326-2333.

Mayer EA (2011) Gut feelings: The emerging biology of gut-"brain communication. Nat Rev Neurosci 12:453-466.

Mönnikes H, Lauer G, Arnold R (1997) Peripheral administration of cholecystokinin activates cfos expression in the locus coeruleus/subcoeruleus nucleus, dorsal vagal complex and paraventricular nucleus via capsaicin-sensitive vagal afferents and CCK-A receptors in the rat. Brain Res 770:277-288.

Morales T, Sawchenko PE (2003) Brainstem prolactin-releasing peptide neurons are sensitive to stress and lactation. Neuroscience 121:771-778. 
Pittig A, Treanor M, LeBeau RT, Craske MG (2018) The role of associative fear and avoidance learning in anxiety disorders: Gaps and directions for future research. Neurosci Biobehav Rev 88:117-140 Available at: https://doi.org/10.1016/j.neubiorev.2018.03.015.

Raybould HE (2010) Gut chemosensing: Interactions between gut endocrine cells and visceral afferents. Auton Neurosci 153:44-46.

Rinaman L (2011) Hindbrain noradrenergic A2 neurons: diverse roles in autonomic, endocrine, cognitive, and behavioral functions. Am J Physiol Regul Integr Comp Physiol 300:R222R235.

Rinaman L, Dzmura V (2007) Experimental dissociation of neural circuits underlying conditioned avoidance and hypophagic responses to lithium chloride. Am J Physiol - Regul Integr Comp Physiol 293:1495-1503.

Ruxton GD (2006) The unequal variance t-test is an underused alternative to Student's t-test and the Mann-Whitney U test. Behav Ecol 17:688-690.

Shapiro RE, Miselis RR (1985) The central organization of the vagus nerve innervating the stomach of the rat. J Comp Neurol 238:473-488.

Spencer SJ, Xu L, Clarke MA, Lemus M, Reichenbach A, Geenen B, Kozicz T, Andrews ZB (2012) Ghrelin regulates the hypothalamic-pituitary-adrenal axis and restricts anxiety after acute stress. Biol Psychiatry 72:457-465.

Tschop M, Smiley DL, Heiman ML (2000) Ghrelin induces adiposity in rodents. Nature 407:908-913.

Watson RE, Wiegand SJ, Clough RW, Hoffman GE (1986) Use of cryoprotectant to maintain long-term peptide immunoreactivity and tissue morphology. Peptides 7:155-159.

Zheng H, Rinaman L (2013) Yohimbine anxiogenesis in the elevated plus maze requires hindbrain noradrenergic neurons that target the anterior ventrolateral bed nucleus of the stria terminalis. Eur J Neurosci 37:1340-1349 Available at: https://www.ncbi.nlm.nih.gov/pmc/articles/PMC3624763/pdf/nihms412728.pdf.

Zhu L, Onaka T (2002) Involvement of medullary A2 noradrenergic neurons in the activation of oxytocin neurons after conditioned fear stimuli. Eur J Neurosci 16:2186-2198.

Zigman JM, Jones JE, Lee CE, Saper CB, Elmquist JK (2006) Expression of ghrelin receptor mRNA in the rat and the mouse brain. J Comp Neurol 494:528-548. 